$\mathrm{C}-\mathrm{A} / \mathrm{AP} / \# 116$

October 2003

Stabilization of the Plate Current in Vacuum Tube Power Amplifier Using Cathode Resistor

S. Zheng, J. Keane, M. Meth, R. Spitz, A. Zaltsman

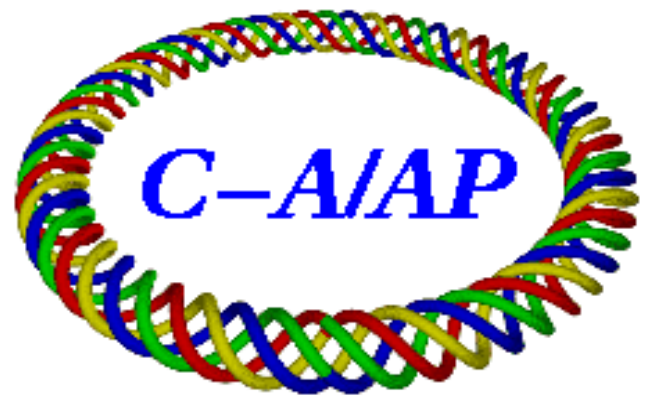

Collider-Accelerator Department Brookhaven National Laboratory

Upton, NY 11973 


\title{
Stabilization of the Plate Current in Vacuum Tube Power Amplifier Using Cathode Resistor
}

\author{
Sanbao Zheng, John Keane, Marvin Meth, Richard Spitz, and Alexander Zaltsman
}

\section{Introduction}

Vacuum tube power amplifier (PA) is a major RF device extensively used in the BNL Collider/Accelerator facilities. Normally the PA operates within a feedback loop to control the cavity gap voltage. In some abnormal situations such as a detuned cavity, the gap voltage is low, and the closed-loop increases the gap voltage by driving the PA harder without considering the increase of plate current. In these cases, the plate current can run away to a dangerous value. Damage to the PA is possible since there is a time delay before the protection device shuts down the PA.

A possible solution to this problem is to introduce another feedback signal derived from the plate current to the closed-loop. This solution, however, is difficult to implement since it requires changing of the system configuration. The plate current is controlled not only by the driving power, but also by the conduction angle of the tube. Thus, for either class $\mathrm{C}$, class $\mathrm{B}$, or class $\mathrm{AB}$ operation, another solution is to increase the magnitude of the control grid bias as the current increases. A cathode resistor Rc (see Fig. 1) is used to realize this method. In this configuration, the cathode resistor essentially forms a negative feedback loop. When the plate current increases, the voltage across Rc increases and the control grid bias becomes more negative, thus the conduction angle becomes smaller and plate current is restrained. The calculations and experimental results for this cathode resistor solution are presented in this note.

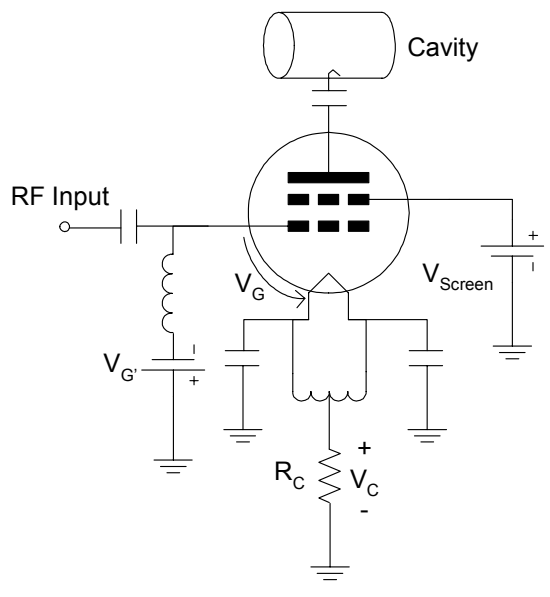

Fig. 1 Circuit configuration.

\section{Approximated calculations}

The driving powers at different plate currents for the $4 \mathrm{CW} 150000$ tetrode tube with a $10 \Omega$ cathode resistor are calculated by an approximate method. Since the control grid bias is a function of the plate current, several iterations may be necessary during the calculation.

Assuming the screen voltage is 1500 volts, initial control grid bias is -400 volts, and the plate voltage is $14 \mathrm{kV}$, from the characteristic curve of the $4 \mathrm{CW} 150000$ the quiescent plate current is about $0.6 \mathrm{~A}$. The characteristic curve also shows a cut-off control grid voltage of about -450 volts. 


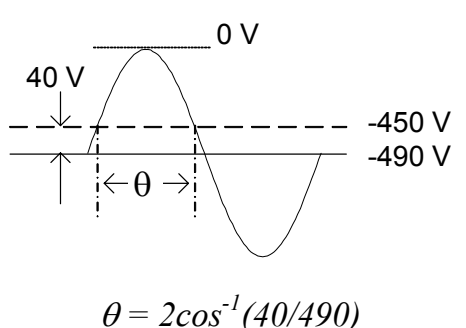

2(a)

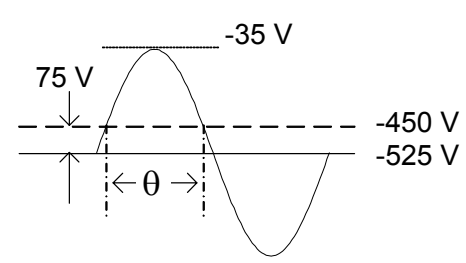

$\theta=2 \cos ^{-1}(75 / 490)$

2(b)

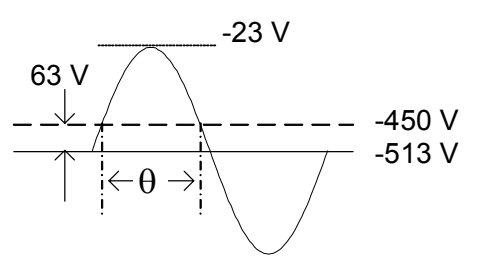

$\theta=2 \cos ^{-1}(63 / 490)$

2(c)

Fig. 2 The grid voltage waveforms used for plate current calculations.

As an initial calculation, assume the plate current $\mathrm{I}_{\mathrm{dc}}$ to be $9 \mathrm{~A}$, and thus the actual bias $\mathrm{V}_{\mathrm{G}}$ is $\mathrm{V}_{\mathrm{G}^{\prime}}, \mathrm{V}_{\mathrm{C}}=-490$ volts. Also assume an input voltage $\mathrm{V}_{\mathrm{g}}$ with a magnitude of 490 volts, thus the maximum grid voltage is $\mathrm{V}_{\mathrm{g}}+\mathrm{V}_{\mathrm{G}}=0$. From the characteristic curve, the peak plate current $I_{\mathrm{m}}$ is $53 \mathrm{~A}$. Under these assumptions, the grid voltage is shown in Fig. 2(a), where $\theta$ is the conduction angle of the tube. The value of $\theta$ can be calculated from the quantities given in the figure. Once this angle is known, a plot given in Page 447, reference [1] can be used to find the ratio of the dc plate current to the peak plate current $\beta=\mathrm{I}_{\mathrm{dc}} / \mathrm{I}_{\mathrm{m}}$. In the plot there is a parameter $\alpha$, which is usually close to $3 / 2$ for tubes with uniform plane electrodes. For the tube used in this note, $\alpha=2$ was found to be a reasonable value [2].

From Fig. 2(a), the conduction angle is found to be $170.6^{\circ}$. $\beta$ is found to be 0.235 from the plot given in [1]. Therefore, $\mathrm{I}_{\mathrm{dc}}=\beta \mathrm{I}_{\mathrm{m}}=12.5 \mathrm{~A}$, which is bigger than the initial assumption.

For the next iteration, assume $\mathrm{I}_{\mathrm{dc}}=12.5 \mathrm{~A}$ (the result from the first calculation). Repeat the above calculations. The resulted control grid voltage is shown in Fig. 2(b), and $\mathrm{I}_{\mathrm{dc}}$ is found to be $10.1 \mathrm{~A}$.

Interpolating Idc by averaging the above two results one obtains a new plate current of $11.3 \mathrm{~A}$. As a check, the calculation given in iteration 1 is performed again. It turns out that the result from this calculation agrees with the interpolated one.

The results of the calculation are given in table 1. So far only one point of the operation is calculated. Continuing this calculation with different values of $\mathrm{V}_{\mathrm{G}}$, the results for other points are also obtained. Assuming a grid load of $200 \Omega$, the grid voltages are converted to driving power, and the final results are summarized in table 2 .

Table 1. Summarization of the calculations with a grid swing of 490 Volts.

\begin{tabular}{|c|c|c|c|c|c|}
\hline Iteration \# & Assumed $\mathrm{I}_{\mathrm{p}}(\mathrm{A})$ & $\mathrm{I}_{\mathrm{m}}(\mathrm{A})$ & $\theta\left(^{\circ}\right)$ & $\beta$ & $\mathrm{I}_{\mathrm{dc}}(\mathrm{A})$ \\
\hline 1 & 9 & 53 & 170.6 & 0.235 & 12.5 \\
\hline 2 & 12.5 & 45 & 162.4 & 0.225 & 10.1 \\
\hline 3 & 11.3 & 50 & 164.7 & 0.226 & 11.3 \\
\hline
\end{tabular}

Table 2. Calculated plate currents at different driving powers.

\begin{tabular}{|c|c|c|c|c|c|c|c|c|}
\hline Drive power (W) & 12.3 & 42.3 & 90.3 & 156.3 & 240.3 & 342.3 & 462.3 & 600.3 \\
\hline Plate current (A) & 0.94 & 1.8 & 2.8 & 3.9 & 5.7 & 6.9 & 9.5 & 11.3 \\
\hline
\end{tabular}


Using the results given in table 2, the operating characteristic given by the driving power vs. plate current is plotted in Fig. 3.

\section{Experimental results}

Cathode resistors at different values are experimented in the RHIC $28 \mathrm{MHz}$ PA, with a dummy load. The dummy load, which is comprised of a transmission line and a pure resistor, provide a load resistance of about 900 $\Omega$ at resonance. The experimental results are shown in Fig. 4 to Fig. 5. Fig. 4 gives the comparisons between the results with a $10 \Omega$ cathode resistor and with no cathode resistor. As expected, the driving power needed is much higher when the cathode resistor is added to the PA, especially when the plate current is

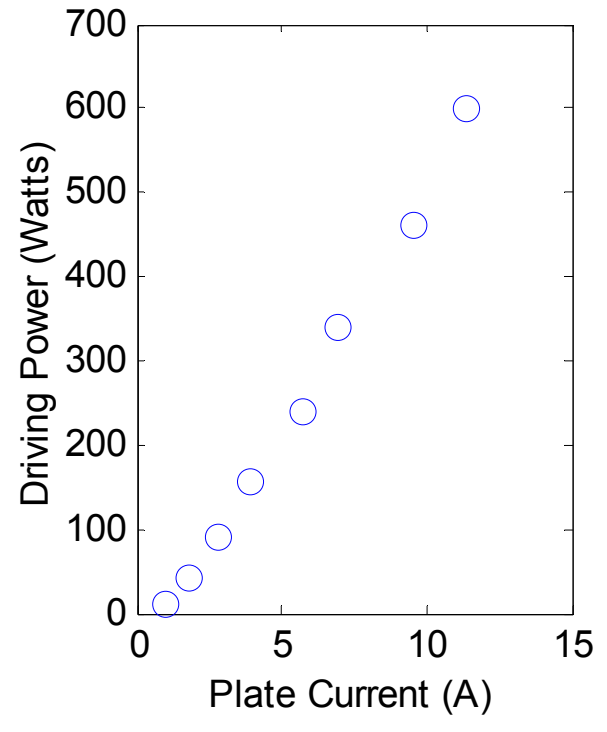

Fig. 3 Calculation result. high. Fig. 5 gives a comparison of the results with different cathode resistors. It is evident that the driving power increases with the increase of the cathode resistance value. The efficiency of the PA also increases with the cathode resistance. This is because the PA operates closer to class $\mathrm{C}$ with the increase of the cathode resistance.

\section{Summary}

A cathode resistor can be used in a high power vacuum PA operating in class $A B, B$, or $\mathrm{C}$ to restrain the plate current from running away. The higher the cathode resistance, the better the current restraining effect. Calculations and experimental results are presented in this note. Experimental results show that, for different values of the cathode resistor, the output power of the PA is essentially the same for the same plate current.

Compromise needs to be made while choosing the cathode resistance since it changes the normal operation of the PA in two aspects: 1) Higher driving power is necessary for the same output; 2) The PA goes to class $\mathrm{C}$ operation and bigger harmonics may be produced.

\section{References}

[1] F. D. Terman, "Radio engineer's handbook," First edition, McGraw-hill book Company, 1943.

[2] S. Zheng and J. Keane, "Modeling and Simulation of the Power Amplifier for the RHIC $28 \mathrm{MHz}$ Accelerating Cavity," BNL note C-A/AP/97, 05/03. 

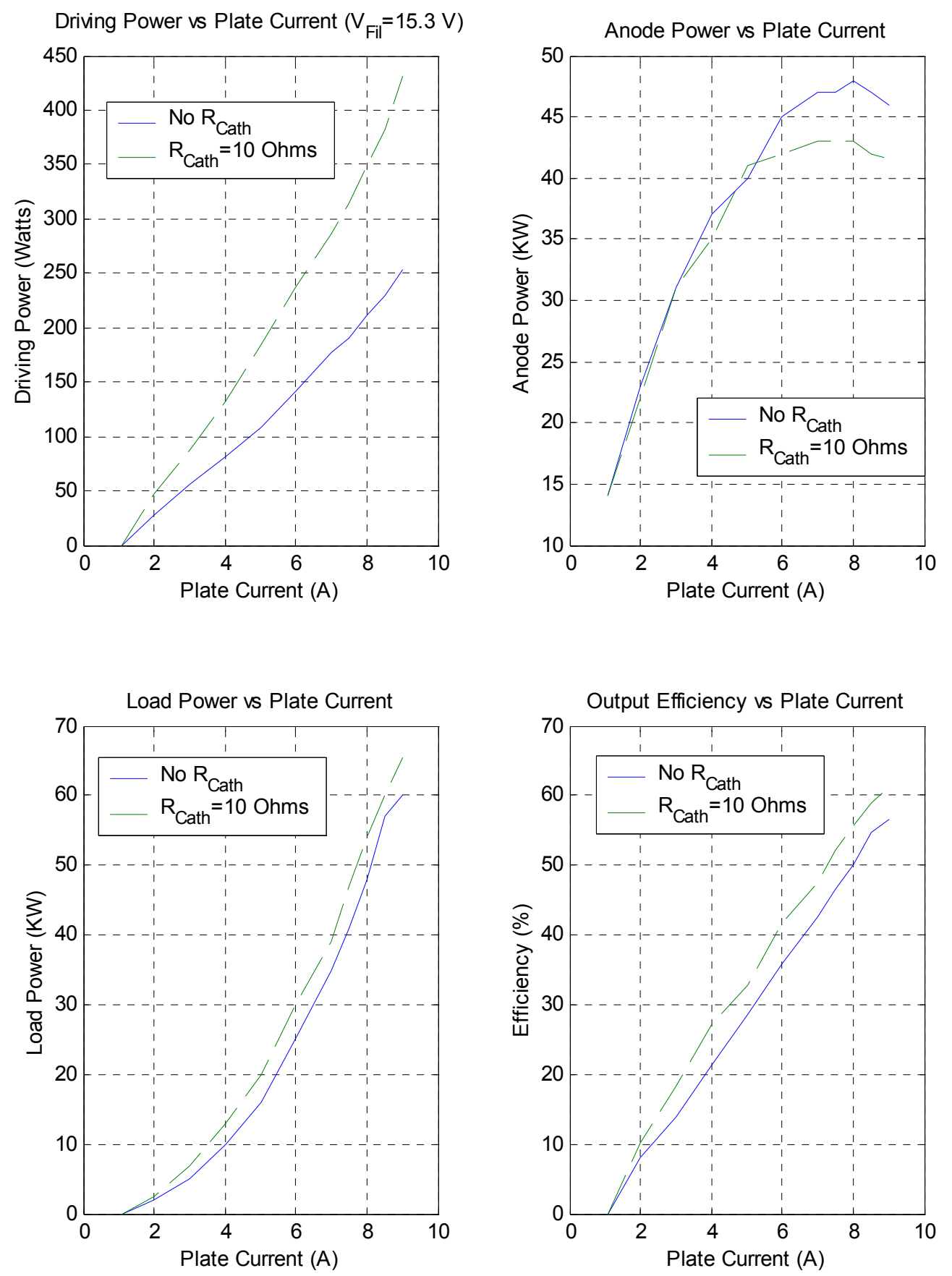

Fig.4 The experimental results of the PA with and without the cathode resistor. 

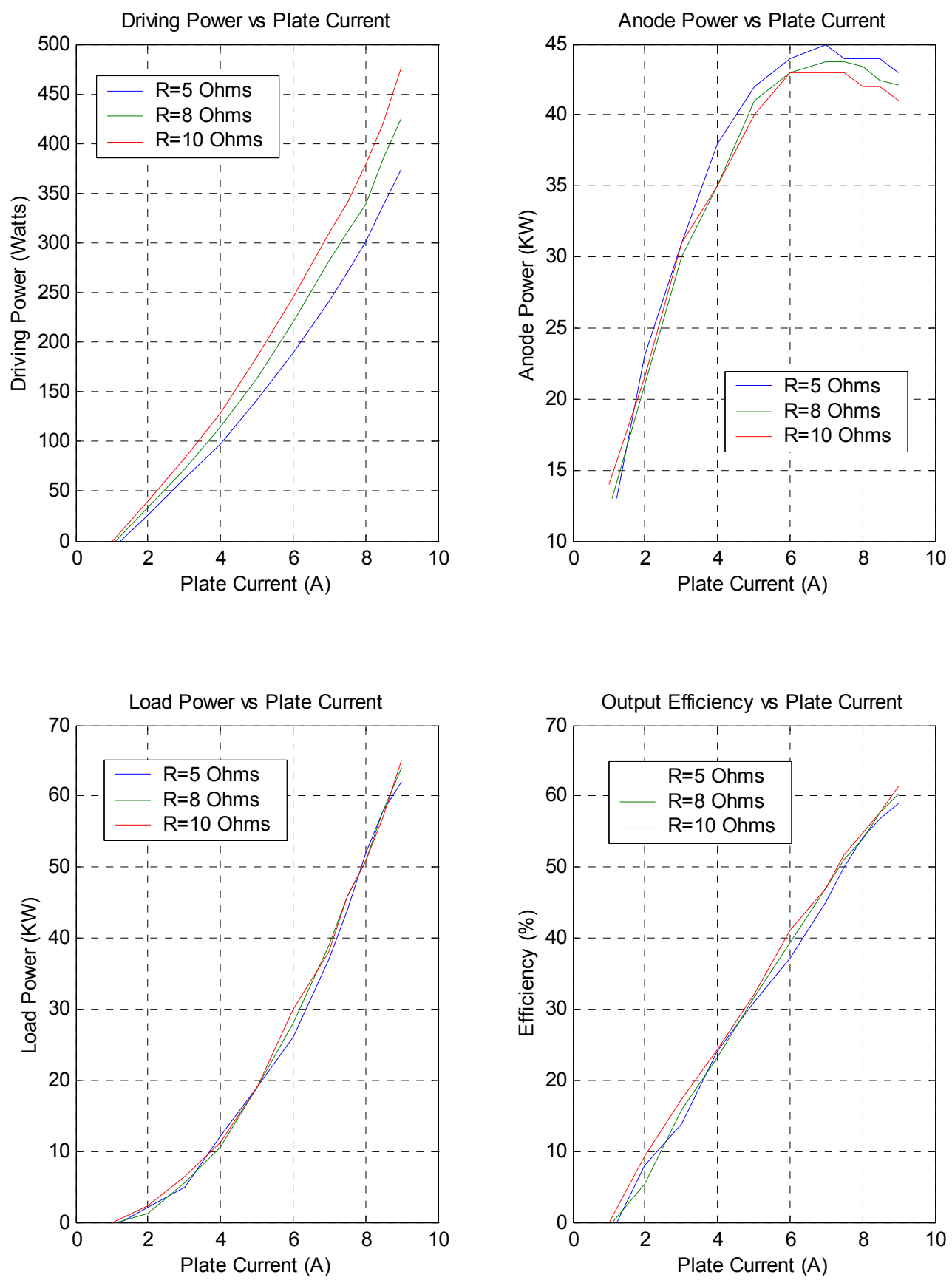

Fig.5 The experimental results of the PA with different cathode resistors. 